\title{
Nuclear Cardiology: The Basics-How to Set Up and Maintain a Laboratory
}

\author{
F.J.Th. Wackers, W. Bruni, and B.L. Zaret \\ Totowa, NJ: Humana Press, 2008, 436 pages, $\$ 99.50$
}

Nuclear cardiology accounts for nearly half of all radionuclide procedures. This text is the second edition of a guide to setting up a free-standing outpatient nuclear cardiology laboratory. A major strength of the book is that the detailed descriptions of the lab, staffing, and practical points of patient and data management are also applicable to general nuclear medicine practices.

The text is written by physician and technologist experts in nuclear cardiology, with about 100 person-years of aggregate field experience among them. The material is clearly presented in a "how to" and "what for" fashion. The reader is guided through the process of designing, equipping, staffing, and working in nuclear cardiology. Information is presented with sufficient detail to allow a reader to make a checklist of items and activities that must be done to get the facility running. For example, in chapter 1 the authors start with basic issues necessary to start the facility. They cover room dimensions (imaging rooms, stress rooms, injection room, patient preparation area, reception or waiting rooms and toilet facilities, radiopharmacy, reading and interpretation area, storage area, staff area, and office space), heating, air conditioning, floor loading, and electrical power. They describe various pieces of equipment necessary for operation of the facility, including imaging devices, acquisition and analysis computer systems, dose calibrators, radiation safety monitoring devices, and personal dosimeters. The authors then discuss the training and experience required to qualify as an imaging technologist, ECG or stress technologist, medical staffer interpreting examinations, or radiation safety officer working in the facility.

All of this is covered in the first chapter. The remaining 23 chapters are equally rewarding, with nuts-and-bolts information on stress testing, imaging, data processing and analysis, reporting, record keeping, laboratory accreditation, and billing.
The text is well illustrated, primarily with black and white renditions of color images. To allow the reader to appreciate the data in color, the authors, thoughtfully, include a CD with important illustrations available for cinematic display in full color.

Major strengths of this text include detailed procedures, guidelines, and forms for patient preparation, stress test acquisition, SPECT acquisition and processing, planar and PET myocardial perfusion imaging, and equilibrium blood pool imaging. The authors include information describing appropriate techniques for display and analysis of nuclear cardiology data, sample reports, quality assurance procedures, and protocols and policies for the laboratory. Although many of the recommendations are derived from the authors' experience at Yale University, information from position papers published by the American College of Cardiology, the American Heart Association, and the American Society of Nuclear Cardiology are included. These documents and references provide the reader with a detailed rationale for each procedure.

The disk provides a particularly rich source of images and technical pointers for acquisition and analysis of myocardial perfusion imaging, as well as the section on quality control.

Overall, this text should be useful for individuals completing their training in nuclear cardiology either through cardiology or nuclear medicine residencies.

One suggestion, should the authors decide to have a third edition, is that the $\mathrm{CD}$ should have an accompanying soundtrack such that the reader has the complete book on $\mathrm{CD}$ rather than being forced to read the book and then refer to specific images on the $\mathrm{CD}$. 